ARTICLE

\title{
Spatiotemporally programmable cascade hybridization of hairpin DNA in polymeric nanoframework for precise siRNA delivery
}

Feng $\mathrm{Li}^{1,4}$, Wenting $\mathrm{Yu}^{1,4}$, Jiaojiao Zhang ${ }^{1}$, Yuhang Dong ${ }^{1}$, Xiaohui Ding ${ }^{1}$, Xinhua Ruan ${ }^{2}, \mathrm{Zi} \mathrm{Gu}{ }^{3}$ \&

Dayong Yang $\mathbb{1}^{1 \times}$

DNA nanostructures have been demonstrated as promising carriers for gene delivery. In the carrier design, spatiotemporally programmable assembly of DNA under nanoconfinement is important but has proven highly challenging due to the complexity-scalability-error of DNA. Herein, a DNA nanotechnology-based strategy via the cascade hybridization chain reaction (HCR) of DNA hairpins in polymeric nanoframework has been developed to achieve spatiotemporally programmable assembly of DNA under nanoconfinement for precise siRNA delivery. The nanoframework is prepared via precipitation polymerization with Acrydite-DNA as cross-linker. The potential energy stored in the loops of DNA hairpins can overcome the steric effect in the nanoframework, which can help initiate cascade HCR of DNA hairpins and achieve efficient siRNA loading. The designer tethering sequence between DNA and RNA guarantees a triphosadenine triggered siRNA release specifically in cellular cytoplasm. Nanoframework provides stability and ease of functionalization, which helps address the complexity-scalability-error of DNA. It is exemplified that the phenylboronate installation on nanoframework enhanced cellular uptake and smoothed the lysosomal escape. Cellular results show that the siRNA loaded nanoframework down-regulated the levels of relevant mRNA and protein. In vivo experiments show significant therapeutic efficacy of using siPLK1 loaded nanoframework to suppress tumor growth.

\footnotetext{
${ }^{1}$ Frontiers Science Center for Synthetic Biology, Key Laboratory of Systems Bioengineering (MOE), School of Chemical Engineering and Technology, Tianjin University, Tianjin, P.R. China. ${ }^{2}$ Department of Cardiac Surgery, Tianjin Union Medical Centre, Tianjin, P.R. China. ${ }^{3}$ School of Chemical Engineering, Australian Centre for NanoMedicine, University of New South Wales, Sydney, NSW, Australia. ${ }^{4}$ These authors contributed equally: Feng Li, Wenting Yu.

凶email: dayong.yang@tju.edu.cn
} 
$\mathrm{n}$ living cells, the physicochemical characteristics of confinement essentially play pivotal roles, such as stabilizing the fold conformation of biomolecules, enhancing biochemical reactivity, affecting biochemical equilibrium, and achieving simultaneous occurrence of multi-bioprocesses in confined spaces ${ }^{1-3}$. Inspired by this unique cellular feature, scientists have made efforts to promote the advances in confinement on non-living chemical systems ${ }^{4}$. For example, confinement effects have been utilized to accelerate reactions or stabilize transition states ${ }^{4-6}$. In particular, confinement effects on the assembly of biomolecules have attracted increasing interest in their great potential in biomedicine ${ }^{5,7-11}$. In the case of deoxyribonucleic acid (DNA), its hybridization and assembly under confined space at nanoscale have been demonstrated to be more favorable than in bulk media because of nanoconfinement entropic effects on chemical equilibrium ${ }^{12,13}$

In recent years, DNA has been used as a versatile buildingblock to assemble functional nanostructures for a wide range of applications due to the unparalleled sequence programmability, molecular recognition, and biological functions ${ }^{14-21}$. In particular, DNA nanostructures have been proven as promising nanocarriers for delivery of nucleic acid drugs ${ }^{17,22,23}$. The isothermal toehold-mediated DNA hybridization chain reaction (HCR) has been developed as an efficient strategy to prepare DNA nanocarriers ${ }^{24,25}$. In HCR, two metastable state species of DNA hairpins can be triggered by an initiator DNA to yield double helix analogous to alternating copolymers ${ }^{21,24,26}$. Spatiotemporally programming assembly of DNA under nanoconfinement via HCR has been proven promising but challenging for the delivery of nucleic acid drugs due to the steric effect and complexity-scalability-error issues ${ }^{27,28}$.

To address the complexity-scalability-error issues, DNA modules have been introduced into synthetic covalent polymers, which are inspired by biological systems that widely adopt incorporating multiple molecular interactions within a system to achieve hierarchical assembly for specific bioprocess ${ }^{29,30}$. For example, Sleiman and coworkers pioneered combining DNA base-pairing with polymer hydrophobic interactions to achieve anisotropic assembly of DNA cages via hydrophobic interactions $^{31}$. Tanja Weil and coworkers constructed polymers grafted DNA origami and realized transformation of the $3 \mathrm{D}$ structural information of DNA origami to polymeric materials ${ }^{32,33}$. Willner and coworkers developed smart hydrogels using DNA-grafted polymers ${ }^{34,35}$. Schulman and coworkers prepared HCR programing high-degree swelling DNA/polymer hybrid hydrogel for the fabrication of soft robots and programmable matters ${ }^{36,37}$. Whereas, DNA assembly under polymeric nanoconfinement has not been explored for the delivery of nucleic acid drugs till now. We envision that achieving programmable assembly of DNA via HCR under polymer-mediated nanoconfinement could be a promising strategy to expand the scalability of DNA for fabricating desirable nanocarriers of nucleic acid drugs.

Herein, the HCR of hairpin DNA in polymeric nanoframework is developed to achieve spatiotemporally programmable assembly of DNA under nanoconfinement for precise siRNA delivery. The polymeric nanoframework with DNA as cross-linker is prepared via precipitation polymerization. By using the potential energy stored in the loops of DNA hairpins to overcome the steric effect under nanoconfinement, the cross-linker DNA in the polymeric nanoframework is designed to initiate the cascade hybridization of two DNA hairpins, thus achieving efficient loading of siRNA in the polymeric nanoframework. Moreover, functional groups such as tumor-targeting phenylboronate are easily decorated on the polymeric nanoframework, which can overcome complexityscalability-error issues of DNA-only nanosystems, improving the in vivo gene delivery efficiency.

\section{Results and discussion}

Molecular design. The DNA cross-linked polymeric nanoframeworks (DPNFs) were prepared via precipitation polymerization of NIPAM (N-isopropylacrylamide), 4-MAPBA ((4-methacrylamidophenyl) boronic acid), Bis (N,N-methylene diacrylamide), and Acrydite-DNA (Fig. 1A). In DPNF, DNA was introduced as cross-linker of the polymer chain and could trigger $\mathrm{HCR}$ of $\mathrm{H} 1$ and $\mathrm{H} 2$ hairpin monomers that were featured with single-stranded toeholds at their $3^{\prime}$ (light blue region) and $5^{\prime}$ ends (dark blue region), respectively (Fig. 1B $)^{26,36}$. The loop region of $\mathrm{H} 1$ contained the complemental sequence of $\mathrm{H} 2$ toehold, and $\mathrm{H} 2$ loop was complementary to $\mathrm{H} 1$ toehold, with the double-stranded stems of $\mathrm{H} 1$ and $\mathrm{H} 2$ being identical (green region). In the absence of trigger DNA, hairpins $\mathrm{H} 1$ and $\mathrm{H} 2$ could store potential energy in loops and coexist metastably; whereas, upon exposure to the trigger DNA (DNA cross-linker in DPNFs), H1 was opened via toehold-mediated hybridization and strand invasion reaction, making the loop sequence freely accessible. The free loop sequence was then attached to the toehold of $\mathrm{H} 2$, thereby initiating a cascade reaction, in which $\mathrm{H} 1$ and $\mathrm{H} 2$ alternately bonded to each other to form long DNA chain in DPNF (Fig. 1B) ${ }^{26,36-39}$. By elaborately designing an ATP (triphosadenine) aptamer overhang on DNA hairpin H2, siRNA with corresponding complementary overhang could be effectively tethered on DNA hairpin $\mathrm{H} 2$ and inserted into the DPNFs with HCR proceeding (Fig. 1B). In response to ATP that was abundant in cells, controlled siRNA release specifically in cellular cytoplasm can be achieved (Fig. 1C) ${ }^{40-42}$. Phenylboronate was introduced onto the DPNFs to enhance cellular uptake via actively recognizing the over-expressed sialic acid residues on tumor cell membrane, and to smooth the lysosomal escape by virtue of acidic $\mathrm{pH}$-responsive transformation from a negatively charged tetravalent hydrophilic form to an uncharged trivalent hydrophobic form ${ }^{43}$. The subtle integration of DNA and synthetic polymer in the DPNF system was anticipated to help achieve in vivo precise siRNA delivery.

Preparation of DPNFs and HCR of DNA hairpins in DPNF for siRNA loading. For the preparation of DPNFs, the molar proportion of 4-MAPBA in the monomers was optimized firstly (Supplementary Figs. 1 and 2) ${ }^{44}$. The molar proportion of 4 MAPBA was varied from 1 to $5 \%$. Scanning electron microscope (SEM) images and dynamic light scattering (DLS) analysis showed that the molar proportion of 4-MAPBA $4 \%$ led to the generation of nanoparticles with minimal diameter of $\sim 250 \mathrm{~nm}$ (Supplementary Fig. 2). Therefore, the molar proportion of 4MAPBA in the monomers was set as $4 \%$ for the preparation of DPNFs. The cross-linker Acrydite-DNA was prepared via selfassembly of two single-stranded DNA C1 and C2 (Supplementary Table 1), which were partially complementary to each other. The concentrations of Acrydite-DNA were set as 5, 10, and $20 \mu \mathrm{M}$, yielding DPNFs denoted as DPNF-5, DPNF-10, and DPNF-20, respectively (Fig. 2A). The morphology and size of the DPNFs were analyzed by SEM and DLS. SEM images showed that the diameters of DPNF-5 and DPNF-10 were between 400 and $450 \mathrm{~nm}$, while the diameter of DPNF-20 was below $200 \mathrm{~nm}$ (Fig. 2B-D). It corresponded to the DLS results in which the average hydrodynamic diameter of DPNF-20 was $266.5 \pm 10.2 \mathrm{~nm}$ and much smaller than that $(\sim 460 \mathrm{~nm})$ of DPNF-5 and DPNF-10 (Fig. 2E). It was speculated that the hydrophilic Acrydite-DNA, as the cross-linker in the DPNF, had significant effect on the polymerization process, cross-linking degree, and nucleation formation of the nanoparticles, and consequently resulted in different sizes of DPNFs. 
A) Formation of DNA cross-linked polymeric nanoframework (DPNF)

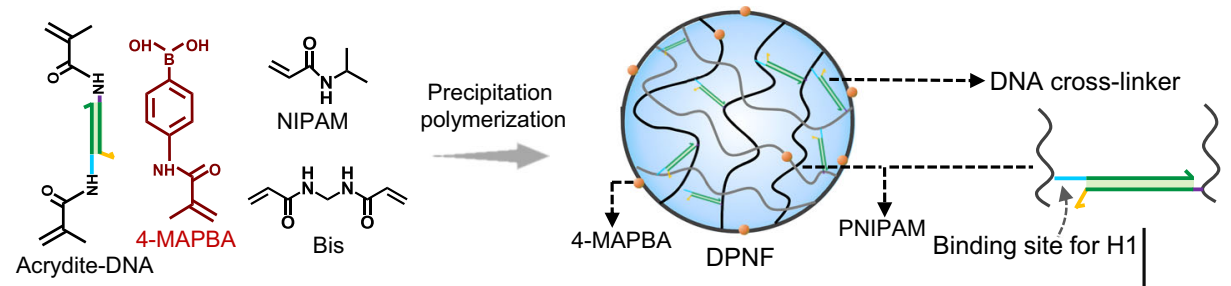

B) DNA cross-linker extension via cascade hybridization of hairpins $\mathrm{H} 1$ and $\mathrm{H} 2$

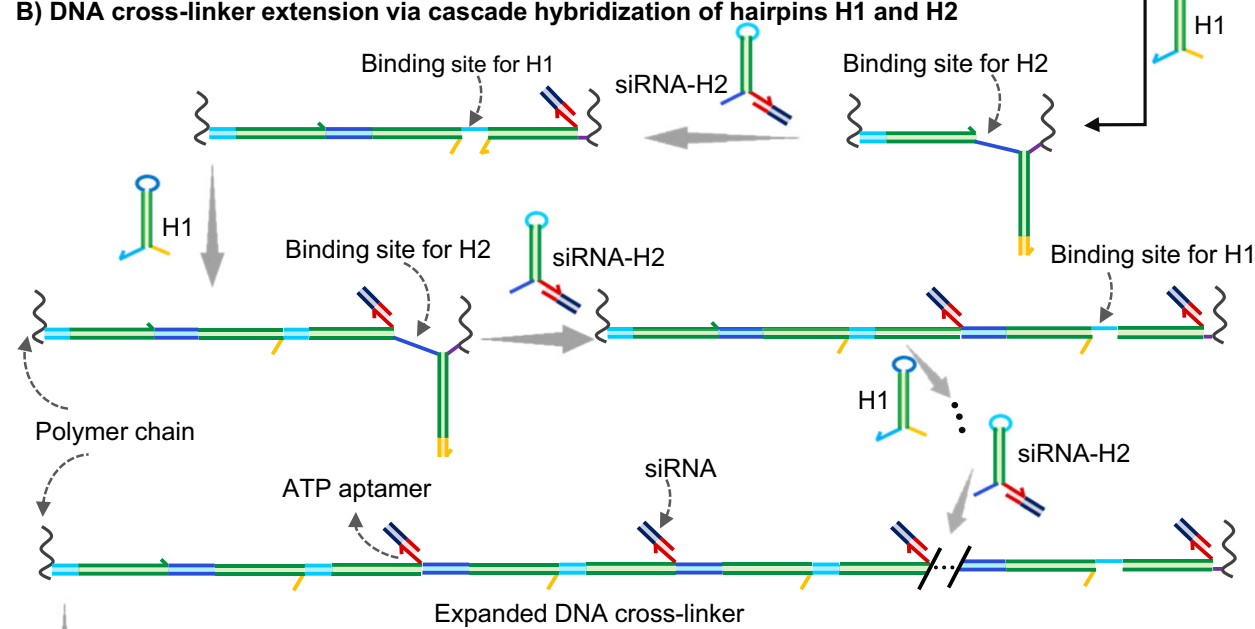

C) ATP triggered siRNA release

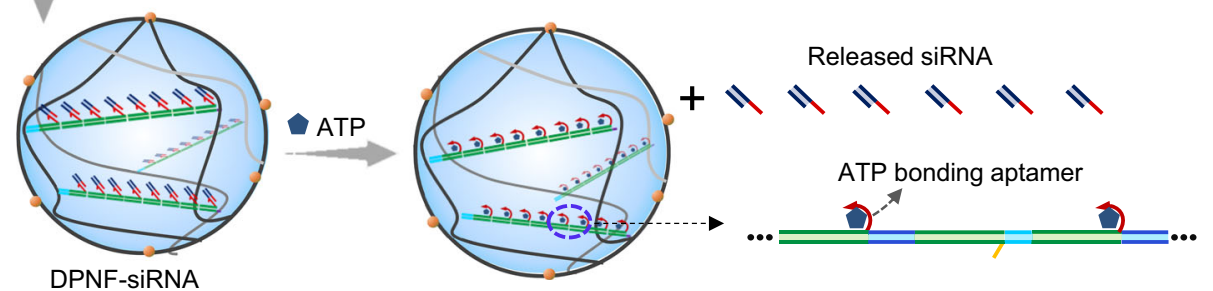

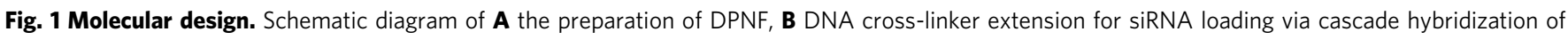
DNA hairpins in DPNFs, and C ATP-triggered release of siRNA from DPNFs. NIPAM, N-isopropylacrylamide; 4-MAPBA, (4-methacrylamidophenyl)

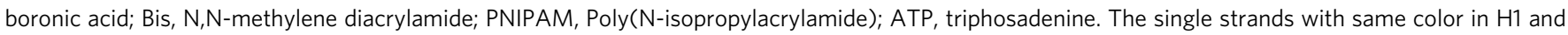
$\mathrm{H} 2$ are complementary sequences.

The two hairpins $\mathrm{H} 1$ and $\mathrm{H} 2$ used for in situ HCR in DPNFs were designed with 18 intramolecular base pairs in stem and 6 base pairs in loop (Supplementary Table 1). Gel electrophoresis analysis was performed and the results showed that they could coexist at $37^{\circ} \mathrm{C}$ (Supplementary Fig. 3). To evaluate the HCR capacity of $\mathrm{H} 1$ and $\mathrm{H} 2$, theoretical calculation was firstly performed and the results showed that the Gibbs free energies of hybridizations between $\mathrm{H} 1$ and $\mathrm{H} 2$ were -55.51 and -54.51 $\mathrm{kcal} / \mathrm{mol}$, respectively, suggesting that $\mathrm{HCR}$ was accessible to $\mathrm{H} 1$ and H2. To further explore the Acrydite-DNA initiating HCR between $\mathrm{H} 1$ and $\mathrm{H} 2$, gel electrophoresis was performed. The gel electrophoresis showed smear bands for a mixture of $\mathrm{H} 1, \mathrm{H} 2$, and Acrydite-DNA, confirming that the hairpins $\mathrm{H} 1$ and $\mathrm{H} 2$ formed long DNA chains in the presence of Acrydite-DNA (Supplementary Fig. 3). Moreover, it was noted that a higher molar ratio of Acrydite-DNA to $\mathrm{H} 1 / \mathrm{H} 2$ resulted in higher hybridization efficiency of $\mathrm{H} 1$ and $\mathrm{H} 2$ but lower molecular weight of DNA chains, which can be explained by the fact that initiator triggered free radical polymerization of small organic monomers.
Further, we explored whether the DNA hairpins $\mathrm{H} 1$ and $\mathrm{H} 2$ could be inserted into the DNA cross-linker in DPNFs via HCR. DPNF-0 (set as control), DPNF-5, DPNF-10, and DPNF-20 were incubated with hairpins $\mathrm{H} 1$ and $\mathrm{H} 2$ (molar ratio of $\mathrm{H} 1$ to $\mathrm{H} 2$ was 1:1) for indicated time period, followed by gel electrophoresis analysis (Fig. 2F-I). Gel electrophoresis images showed that, with prolonging incubation time, the amount of free $\mathrm{H} 1$ and $\mathrm{H} 2$ were sequentially decreased while the DNA trapped in the well was increased for DPNF-5, DPNF-10, and especially DPNF-20. In contrast, for the DPNF-0, the free $\mathrm{H} 1$ and $\mathrm{H} 2$ bands maintained almost identical and little DNA was observed in the well even at incubation time of $24 \mathrm{~h}$. The quantitative analysis of $\mathrm{H} 1$ intensity demonstrated that free $\mathrm{H} 1$ was decreased by $50.1 \%, 58.8 \%$, and $91.0 \%$ for DPNF-5, DPNF-10, and DPNF-20, respectively (Fig. 2J-M). In particular, free $\mathrm{H} 1$ was decreased by $~ 72.2 \%$ within $3 \mathrm{~h}$ for DPNF-20. The results confirmed that the DPNFs could efficiently initiate the HCR of $\mathrm{H} 1$ and $\mathrm{H} 2$ to insert them in DPNFs.

We then examined the evolution of DPNF in morphology and size after HCR using SEM, transmission electron microscopy (TEM), and DLS. SEM images showed that after HCR of H1 and 
A)

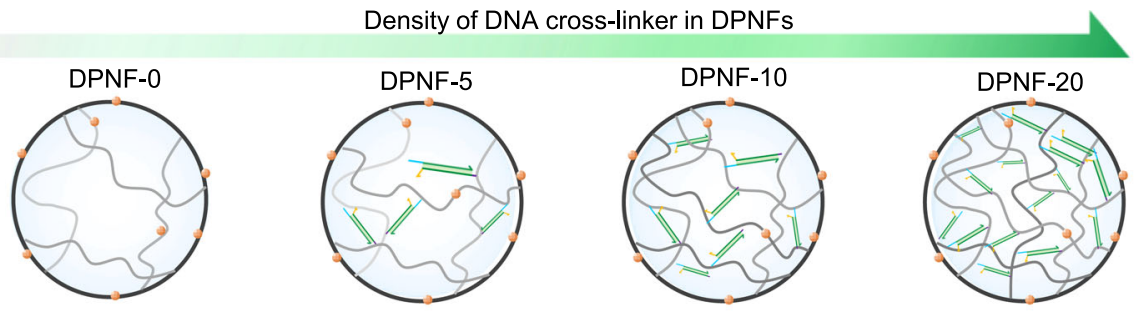

B)

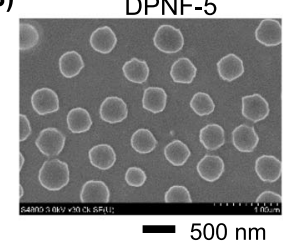

C)

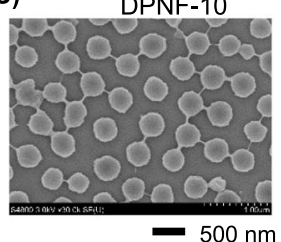

D)

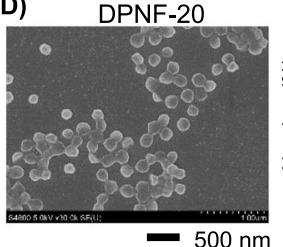

E)

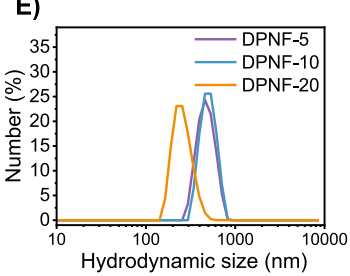

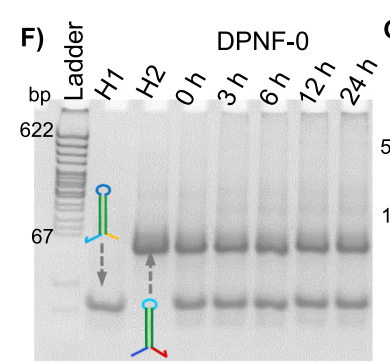

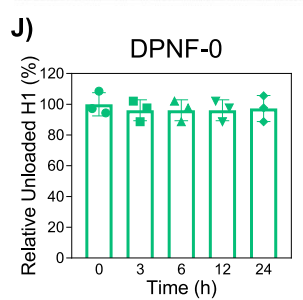

N)

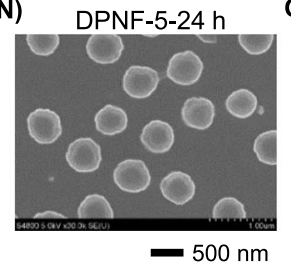

0)

G) ¿্ DPNF-5
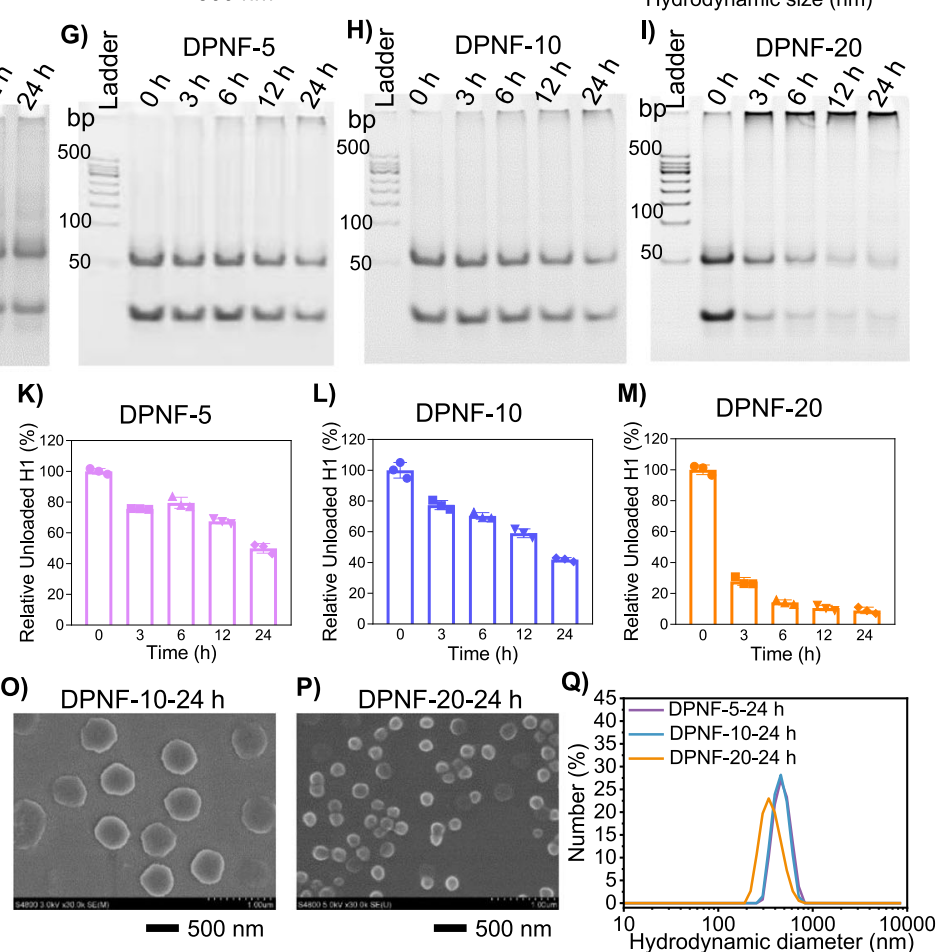

Fig. 2 Preparation of DPNF and in situ HCR of $\mathbf{H 1}$ and H2 in DPNF. A Schematic illustration of DPNFs with varied density of DNA cross-linkers. B-E SEM images and DLS analysis of DPNF-5, DPNF-10, and DPNF-20. F-I Gel electrophoresis analysis of HCR of H1 and H2 in DPNF-0, DPNF-5, DPNF-10, and DPNF-20. The concentration of DPNFs was $4.2 \mathrm{mg} / \mathrm{ml}$. The concentration of $\mathrm{H} 1 / \mathrm{H} 2$ was $3 \mu \mathrm{M}$. J-M Quantitative analysis of unloaded $\mathrm{H} 1$ versus incubation time in gel electrophoresis shown in (F-I) using image J software. Error bars represent s.d. ( $n=3$ independent replicates). N-Q SEM images and DLS analysis of DPNF-5, DPNF-10, and DPNF-20 incubated with $\mathrm{H} 1$ and $\mathrm{H} 2$ for $24 \mathrm{~h}$.

H2 in DPNFs, the DPNF-5, DPNF-10, and DPNF-20 remained well dispersed with a defined spherical structure (Fig. 2N-P and Supplementary Fig. 4). Moreover, the size of all the DPNFs was increased during 24-h incubation: the diameter of DPNF-5 and DPNF-10 was increased from $300-350$ to $450-500 \mathrm{~nm}$, and the diameter of the DPNF-20 was significantly increased from $\sim 155$ to $\sim 180 \mathrm{~nm}$ (Supplementary Figs. 4 and 5). TEM results showed hollow cores in DPNF-5-24, DPNF-20-24, and especially DPNF$10-24$, probably due to the inhomogeneous HCR in DPNFs (Supplementary Fig. 6). DLS results also showed that the hydrodynamic diameter of DPNF-5 and DPNF-10 had a little change, while, the DPNF-20 was significantly increased from $266.5 \pm 10.2$ to $326.1 \pm 35.0 \mathrm{~nm}$ (Fig. 2Q). Zeta potential analysis was performed to explore the change of the surface potential, and the results showed that DPNF-5 and DPNF-10 exhibited slight decrease; in contrast, for DPNF-20, the zeta potential changed from -7.0 to $-13.0 \mathrm{mV}$ (Supplementary Fig. 7).
To explore whether the siRNA could be incorporated into the DPNFs via HCR of DNA hairpins $\mathrm{H} 1$ and $\mathrm{H} 2$, we firstly designed two single-stranded DNA with one sticky end of 10 bases (denoted as ssDNA-10) or 12 bases (denoted as ssDNA-12) that were complementary to the ATP aptamer overhang of $\mathrm{H} 2$ (Supplementary Table 1). The melting temperatures (Tm) for the hybridization of ssDNA-12 and ssDNA-10 with hairpin $\mathrm{H} 2$ were theoretically calculated to be 45.9 and $32{ }^{\circ} \mathrm{C}$, respectively. The connection efficiency with $\mathrm{H} 2$ was evaluated by gel electrophoresis. The gel electrophoresis results demonstrated that when ssDNA-10 and H2 (molar ratio 1:1) were mixed, two separating bands belonging to ssDNA-10 and $\mathrm{H} 2$ were observed, indicating that no hybridization occurred between ssDNA-10 and $\mathrm{H} 2$ (Supplementary Fig. 8A). In contrast, when ssDNA-12 and H2 (molar ratio 1:1) were mixed, a new band with lower mobility than ssDNA1 and $\mathrm{H} 2$ was observed in the gel electrophoresis, demonstrating the successful hybridization between ssDNA-12 


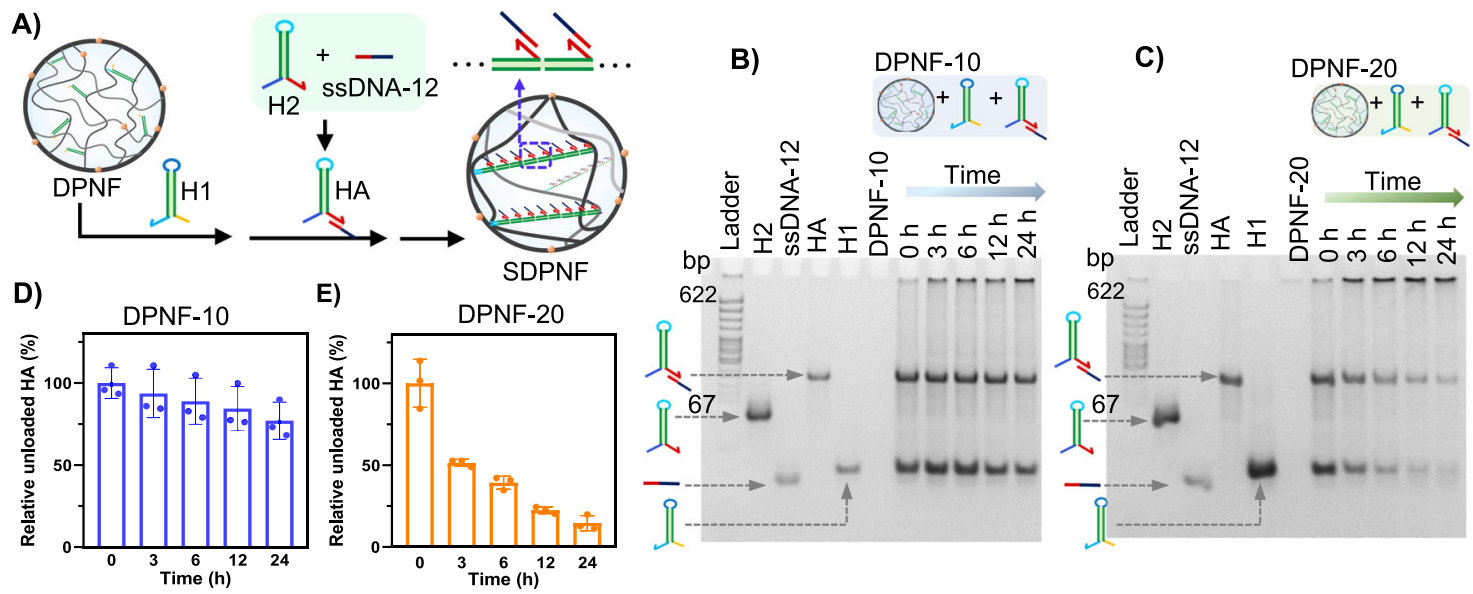

Fig. 3 Programmable assembly of ssDNA-12 in DPNF via HCR of DNA hairpins. A Schematic description of assembling ssDNA-12 in DPNFs: the ssDNA12 was linked with $\mathrm{H} 2$ to form $\mathrm{HA}$ first, and then $\mathrm{H} 1(3 \mu \mathrm{M})$ and $\mathrm{HA}(3 \mu \mathrm{M})$ was co-incubated with DPNFs $(0.42 \mathrm{mg} / \mathrm{ml})$. B Gel electrophoresis analysis of loading of $\mathrm{H} 1(3 \mu \mathrm{M})$ and $\mathrm{HA}(3 \mu \mathrm{M})$ in DPNF-10 or DPNF-20 $(0.42 \mathrm{mg} / \mathrm{ml})$ versus incubation time according to the strategy represented in $(\mathbf{A})$. D, E are quantitative analysis of unloaded HA versus incubation time in $(\mathbf{B})$ and $(\mathbf{C})$, respectively, using image $\mathrm{J}$ software. Error bars represent s.d. $(n=3)$.

and H2 (Supplementary Fig. 8B). Therefore, the ssDNA-12 was employed as the substitute of siRNA for the following assembly and cell imaging study.

Next, two strategies were employed to assemble ssDNA-12 in DPNFs. In the first strategy (Supplementary Fig. 9A), DPNFs were firstly incubated with $\mathrm{H} 1$ and $\mathrm{H} 2$ to insert $\mathrm{H} 2$ into the DPNFs, and then incubated with ssDNA-12. Gel electrophoresis analysis was performed to evaluate the loading efficiency of ssDNA-12. The results showed that, even after 24-h incubation, the band intensity of ssDNA-12 in DPNF-10 and DPNF-20 group exhibited negligible decrease (Supplementary Fig. 9B-E), demonstrating that little ssDNA-12 was loaded in the DPNF-10 and DPNF-20. It was inferred that the steric effect and electrostatic repulsion between DPNFs and polyanion ssDNA-12 impeded their interaction and further hybridization.

Another strategy was the linkage of ssDNA-12 with $\mathrm{H} 2$ to form H2-ssDNA-12 (HA) followed by co-incubation of HA and $\mathrm{H} 1$ with DPNFs (Fig. 3A). The DPNF-10 and DPNF-20 were, respectively, incubated with $\mathrm{H} 1$ and $\mathrm{HA}$. The gel electrophoresis showed that, with prolonging incubation time, the bands belonging to HA and $\mathrm{H} 1$ gradually faded and the DNA trapped in the well was increased, demonstrating the successful assembly of HA in the DPNFs (Fig. 3B, C). After 24-h of incubation, the band intensity of HA was decreased by $22.95 \%$ and $85.36 \%$ for DPNF-10 and DPNF-20, respectively (Fig. 3D, E), confirming that ssDNA-12 was successfully loaded in the DPNFs, and DPNF-20 possessed higher capability for nucleic acid loading than DPNF-10. These results demonstrated that the potential energy stored in the DNA hairpins could overcome the steric effect and electrostatic repulsion to achieve efficient siRNA loading under nanoconfinement.

Phenylboronic acid (PBA) mediating cellular uptake and lysosomal escape of DPNF. Two prerequisites for efficient gene silencing are high nucleic acid drug uptake levels and efficient release in cytoplasm ${ }^{45,46}$. On the membrane of most cancer cells such as MDA-MB-231 (breast cancer cell line) cells, diol containing sialic acid residues is over-expressed. PBA on DPNF-20 could react with sialic acid residues to form annular boronate ester and promote the endocytosis of DPNF-20 (Fig. 4A). The cellular uptake efficiency of DPNF-20 in MDA-MB-231 cells was first evaluated via flow cytometry (FCM) by staining DPNF-20 with Cyanine 5 (Cy5) (Fig. 4B). The FCM analysis results showed significant increase in fluorescence signals in MDA-MB-231 cells with prolonging incubation time, demonstrating continual and effective internalization of Cy5-SDPNF-20. Laser scanning confocal microscope (CLSM) analysis was further performed to explore the mediation function of sialic acid residues in the internalization of DPNF-20. 5-Carboxytetramethylrhodamine (TAMRA) labeled DPNF-20 (denoted as TAMRA-SDPNF-20) was incubated with MDA-MB-231 cells for $6 \mathrm{~h}$, and then CLSM analysis was performed. CLSM images showed significant red fluorescence signals from TAMRA in the cells; whereas, when the cells were pretreated with 4-boronobenzoic acid (BA) that could block the sialic acid receptor before incubation with TAMRASDPNF-20, much weaker fluorescence signals from TAMRA were detected in the cells (Fig. 4C). All these results demonstrated an efficient PBA modulating cellular uptake of TAMRA-SDPNF$20^{47}$.

The unique $\mathrm{pH}$-responsive change of $\mathrm{PBA}$ in hydrophobicity facilitated lysosomal escape of PBA decorated nanoparticles. We envisioned that in the acidic lysosome ( $\mathrm{pH} \sim 5)$, PBA could transform from tetravalent to trivalent, thus resulting in an increase in the hydrophobic PBA fraction and promoting the association of DPNF-20 with lysosomal membranes to realize lysosomal escape ${ }^{43}$. To evaluate the lysosomal escape capability that facilitated by PBA, CLSM analysis of Cy5-DPNF-20 treated MDA-MB-231 cells was performed. The lysosome was stained with LysoTracker Green, and nucleus was stained with DAPI (blue). The overlay of green and red fluorescence demonstrated the Cy5-DPNF-20 that located in lysosomes; the separation of red and green fluorescence demonstrated the Cy5-DPNF-20 that escaped from lysosomes to cytoplasm (Fig. 4D). The Pearson's correlation coefficients of 1 and $2 \mathrm{~h}$ were 0.5396 and 0.5364 quantitatively, demonstrating efficient intracellular lysosomal escape and the dynamic intracellular transport of Cy5-DPNF-20.

ATP-triggered nucleic acid release from DPNFs. Prior to exploring ATP-triggered nucleic acid release from DPNF, we investigated the ATP-responsive property of HA assembled from ssDNA-12 and H2 with gel electrophoresis. The gel electrophoresis showed that when HA was incubated with $5 \mathrm{mM}$ ATP that corresponded to the intracellular ATP concentrations of 5-10 mM, a band of ssDNA-12 was observed (Supplementary Fig. 10), demonstrating that a portion of ssDNA-12 quickly dissociated from HA. Whereas, still a portion of ssDNA-12 did not dissociate from the HA after $8 \mathrm{~h}$. It was inferred that the competitive binding between ATP and ssDNA-12 with ATP aptamer 

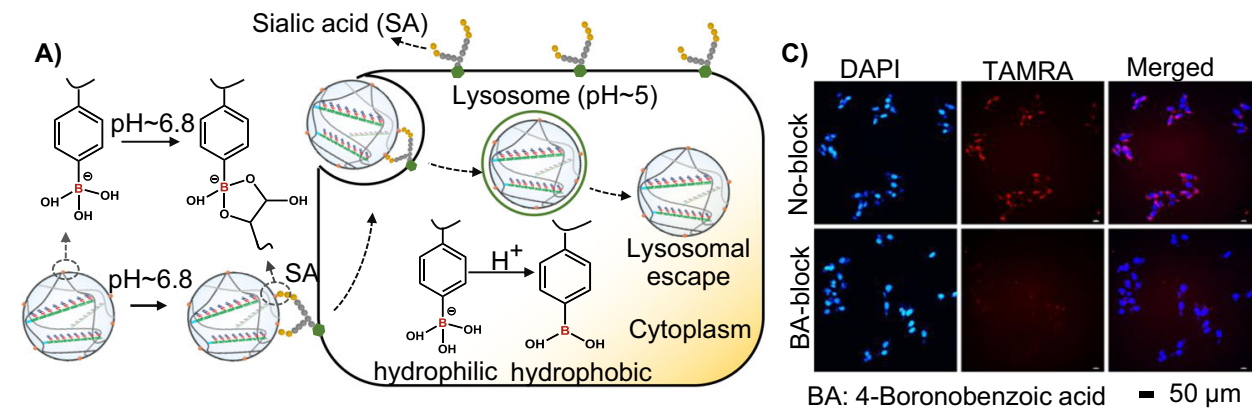

B)

D) Cy5 Lyso-Tracker DAPI

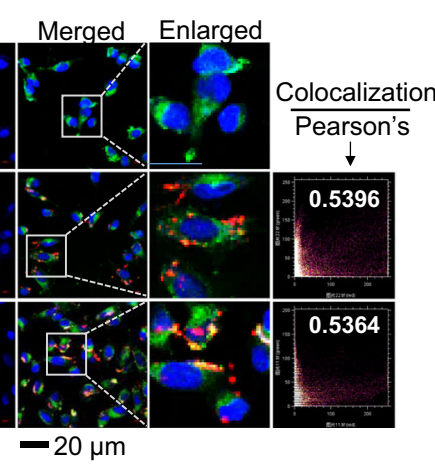

Fig. 4 PBA mediating cellular uptake and lysosomal escape of DPNF. A Schematic illustration of PBA mediating cellular uptake and lysosomal escape of DPNFs. B Flow cytometry analysis of cellular uptake of Cy5-SDPNF-20 $(420 \mu \mathrm{g} / \mathrm{ml})$ by MBA-MD-231 cells at different incubation time. C CLSM images of TAMRA-SDPNF-20 treated MBA-MD-231. BA-block group, cells were pretreated with 4-boronobenzoic acid (BA, $0.2 \mathrm{mg} / \mathrm{ml}$ ) and then incubated with TAMRA-SDPNF-20 (420 $\mu \mathrm{g} / \mathrm{ml})$; No-block group, cells were incubated with TAMRA-DPNF-20 without BA pretreatment. D CLSM images of MBA-MD231 cells incubated with Cy5-DPNF-20 $(420 \mu \mathrm{g} / \mathrm{ml})$ at different time points. Cellular nuclei (blue) were stained by 4',6-diamidino-2-phenylindole (DAPI) and lysosomes were stained with LysoTracker Green.
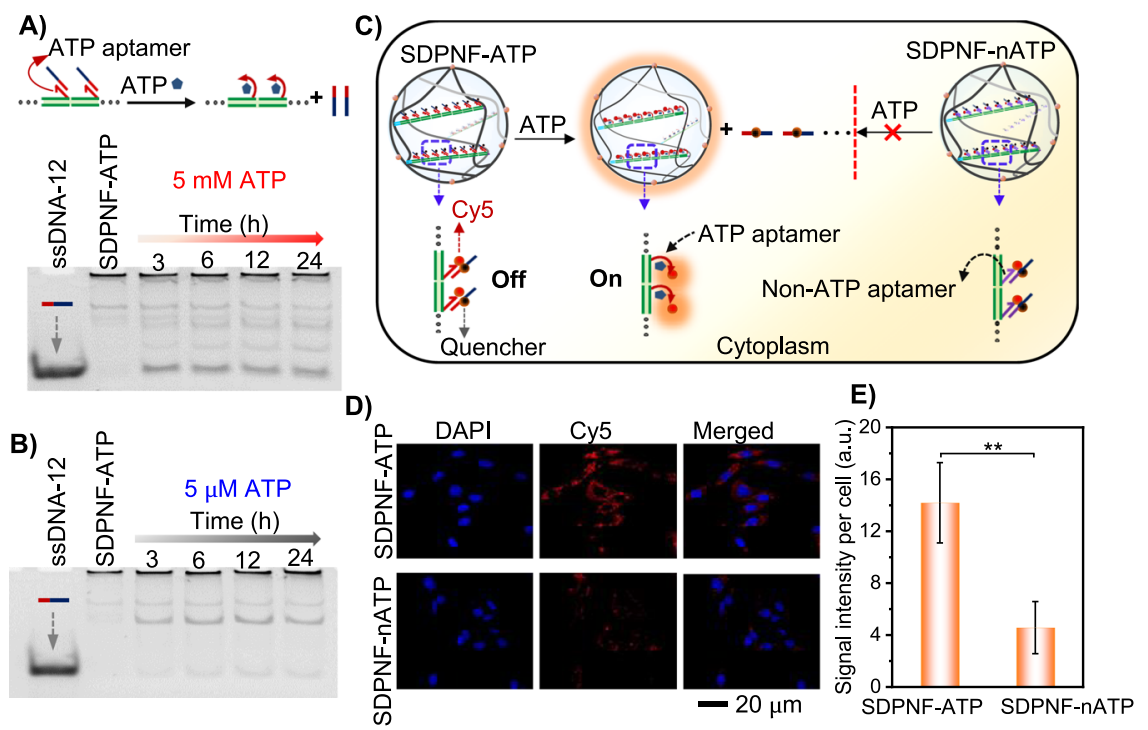

Fig. 5 ATP triggered ssDNA-12 release from ATP-responsive DPNF (SDPNF-ATP). A, B ATP-triggered ssDNA-12 release profiles of SDPNF-ATP in which ssDNA-12 was linked via ATP aptamer sequence. The concentrations of SDPNF-ATP and ssDNA-12 were $4.2 \mathrm{mg} / \mathrm{ml}$ and $3 \mu \mathrm{M}$, respectively. C Schematic illustration of exploration on ATP-triggered ssDNA-12 release from SDPNF-ATP in cytoplasm via FRET. The 3' overhang of H2 was modified with Cy5 as a fluorescent label and the middle base in SSDNA-12 was modified with BHQ2 as a quencher. SDPNF-nATP, the SSDNA-12 was linked via nonATP (nATP) aptamer sequence. D CLSM images of MDA-MB-231 cells treated with SDPNF-ATP (420 $\mu \mathrm{g} / \mathrm{ml})$ and SDPNF-nATP (420 $\mu \mathrm{g} / \mathrm{ml})$ for $6 \mathrm{~h}$, respectively. E Quantitative analysis of fluorescence intensity per cell in SDPNF-ATP group and SDPNF-nATP group. Error bars represent s.d. $(n=20$ cells), ${ }^{\star \star} p<0.01$.

in $\mathrm{H} 2$ was responsible for the incomplete release of ssDNA-12. When ssDNA-12-loaded ATP-responsive DPNF-20 (denoted as SDPNF-ATP) was incubated with $5 \mathrm{mM}$ ATP, a quick release of ssDNA-12 was observed; whereas, no more ssDNA-12 was released even the incubation time was prolonged to $12 \mathrm{~h}$ depending on the gray change of ssDNA-12 band (Fig. 5A). The steric effect and electrostatic repulsion between DPNFs and released ssDNA-12 impeded their re-hybridization with DPNFs according to the results in Supplementary Fig. 10. Therefore, it was inferred almost all the ssDNA-12 was released from SDPNF- 
ATP within $3 \mathrm{~h}$. However, when the concentration of ATP was decreased to $5 \mu \mathrm{M}$ that was higher than the ATP level in plasma $(100 \mathrm{nM})$, only very slight bands belonging to free ssDNA-12 were observed (Fig. 5B), demonstrating that a very small portion of ssDNA-12 was released from SDPNF-ATP. Furthermore, when the ATP aptamer overhang of $\mathrm{H} 2$ was replaced with a scramble DNA sequence (non-ATP aptamer, Supplementary Table 1), no ssDNA-12 was released in $5 \mathrm{mM}$ ATP from SDPNF-nATP in which the ssDNA-12 was linked via non-ATP (nATP) aptamer sequence (Supplementary Fig. 11). These results demonstrated the sensitive and specific release capacity of DPNF-20 in response to intracellular ATP.

Furthermore, the ATP-triggered ssDNA-12 release from SDPNF-ATP inside cells was confirmed by using fluorescence resonance energy transfer (FRET). The $3^{\prime}$ overhang of $\mathrm{H} 2$ was modified with Cy5 as a fluorescent label and the middle base in ssDNA-12 was modified with black hole quencher BHQ2. In SDPNF, the fluorescence of Cy5 was quenched due to the FRET effect; when ssDNA-12 was released, the fluorescence of Cy5 would be recovered (Fig. 5C). The MDA-MB-231 cells were incubated with labeled SDPNF-ATP and then imaged with CLSM. The labeled SDPNF-nATP was set as control. CLSM images showed significant red fluorescence signals from Cy5 in the SDPNF-ATP treated cells, confirming the effective release of ssDNA-12 in the cells (Fig. 5D). Whereas, the lower level of Cy5 fluorescence signals was detected in the DPNF-nATP treated cells. Quantitative analysis showed $\sim 3.5$ times stronger fluorescence signals per cell in the SDPNF-ATP group than that of the SDPNF-nATP group (Fig. 5E). The results demonstrated that the ATP-responsive capacity facilitated the quick release of the target nucleic acid drugs in cytoplasm, which was important for the effective gene silencing effect.

Gene silencing effect of siRNA-loaded DPNF-20 in vitro. The gene silencing efficiency of siRNA-loaded DPNF-20 was evaluated in MBA-MD-231 cells by using actin gene as the target gene. The silence effect in terms of expression of cellular actin cytoskeletons was confirmed with Lipo3000 as transfection reagent (Supplementary Fig. 12). MBA-MD-231 cells were incubated with different formulations of siActin, i.e., naked siActin, DPNF-siScram (DPNF-20 loaded with scramble RNA sequence), DPNF-nATP-siActin (siActin was linked with non-ATP aptamer in DPNF), and DPNF-ATP-siActin (siActin was linked with ATP aptamer in DPNF) at the same dose $(300 \mathrm{nM})$ of siActin for $6 \mathrm{~h}$. After another 44-h incubation, the cellular actin cytoskeletons were stained with phalloidin-TRITC (red fluorescence) and imaged with fluorescence microscope. Red fluorescence signals of the naked siActin group and the DPNF-siScram group are comparable to the blank group. However, the DPNF-nATPsiActin and DPNF-ATP-siActin treated groups showed weaker signals than the blank group, and the latter group was even weaker than the former one. The results suggested that the DPNF-nATP-siActin and DPNF-ATP-siActin could downregulate the expression of cytoskeletons (Fig. 6A). The cytoskeleton expression per cell was further quantitatively analyzed according to the fluorescence intensities. The results indicated that exposure to naked siActin or DPNF-siScram did not affect the expression of cytoskeletons (Fig. 6B). In contrast, in cells treated, respectively, with DPNF-nATP-siActin and DPNF-ATPsiActin, the cytoskeletons were significantly reduced by $28.58 \%$ and $46.68 \%$, respectively.

In vitro therapeutic effect of siRNA-loaded DPNF-20 was then evaluated by using polo-like kinase 1 (PLK1), a key regulator of cellular proliferation that over-expressed in many malignant cells such as MBA-MD-231 cells, as an oncogenic target for RNA interfere therapy. MDA-MB-231 cells were treated with different formulations of anti-PLK1 siRNAs in culture for $6 \mathrm{~h}$. After another 44-h incubation, the PLK1 mRNA and protein expression were measured by real-time quantitative polymerase chain reaction (RT-qPCR) and western blotting, respectively. RT-qPCR results showed that naked siPLK1 treated group and DPNFsiScram treatment did not change the level of PLK1 mRNA compared to the blank group. Notably, DPNF-nATP-siPLK1 group and DPNF-ATP-siPLK1 group showed $30.53 \%$ and $44.36 \%$ downregulation of PLK1 mRNA, respectively (Fig. 6C). Consistently, the western blot results showed that, compared with blank groups, no significant downregulation of PLK1 protein was detected in the naked siPLK1 group or DPNF-siScram treated groups (Fig. 6D). However, for DPNF-nATP-siPLK1 and DPNFATP-siPLK1-200 treated groups, the expression of PLK1 protein was, respectively, reduced by $\sim 48 \%$ and $\sim 80 \%$ as shown by the band intensity. Furthermore, it is worth to note that even when the concentration of siPLK1 was reduced to $200 \mathrm{nM}$ in the DPNF-ATP-siPLK1-200 treated group, the expression of PLK1 protein was reduced by $\sim 63 \%$, which was much higher than that of DPNF-nATP-siPLK1 treated group in $300 \mathrm{nM}$, thus confirming that the rapid release of siRNA in the cytoplasm was a vital factor for effective gene silencing.

Biocompatibility, tumor targeting, and antitumor effect in vivo. In vitro biocompatibility test of DPNF-ssDNA-12 was performed with standard MTT (3-(4,5-dimethylthiazol-2-yl)-2,5diphenyltetrazolium bromide) assay. MDA-MB-231 cells were incubated with DPNF-20 in varied concentrations for $24 \mathrm{~h}$, and then the cell viability was tested via MTT assay. The MTT results showed that the cells remained satisfactory viability that was higher than $80 \%$, demonstrating the good biocompatibility of DPNF-20 (Supplementary Fig. 13).

To assess the in vivo biosafety of the DPNFs as siRNA delivery vector, we examined the effects of the DPNF-20 on healthy BALB/ $c$ mice with normal immunity. Two groups were studied including saline-treated group as control and DPNF-20-treated group $(n=$ 3 ). On days 1,4 , and 7 , drug intravenous injections were carried out and body weight was measured; on day 10 the mice were euthanized, and the serum and major organs were collected. The body weight curves showed negligible change in the DPNF-20treated group compared to the saline (Supplementary Fig. 14A). To study potential changes in organ morphology, postmortem histopathology of the heart, liver, and kidney was analyzed, and no obvious morphological changes were observed (Supplementary Fig. 14B). To further evaluate the potential effects of the DPNFs on the functions of the heart, liver, and kidney, relative serum biochemical index analysis was performed. The collected biochemical indexes showed negligible difference between DPNF-20treated group and saline-treated group (Supplementary Fig. 14C). It was proposed that the biodegradability property of the crosslinker DNA facilitated the final degradation of DPNFs in vivo. After the enzymatic degradation of cross-linker DNA, the structure of the carrier materials disassembled to release the polymer chains, which could be degraded to oligomers by reactive oxygen species or enzymes in living cells.

Prior to exploring in vivo targeting ability of DPNF-20, the stability of the DPNF-20 was evaluated by incubating DPNF-20 in FBS-containing cell culture medium via polyacrylamide gel electrophoresis ${ }^{48,49}$. It was noteworthy that, almost all DNA was stuck in the well of the gel within $6 \mathrm{~h}$, and there were still more than $60 \%$ DNA was stuck in the well according to the gray analysis of the DNA bands, thus indicating that SDPNF-20 could significantly prevent nuclease attack and kept stability in physiological conditions (Supplementary Fig. 15). 

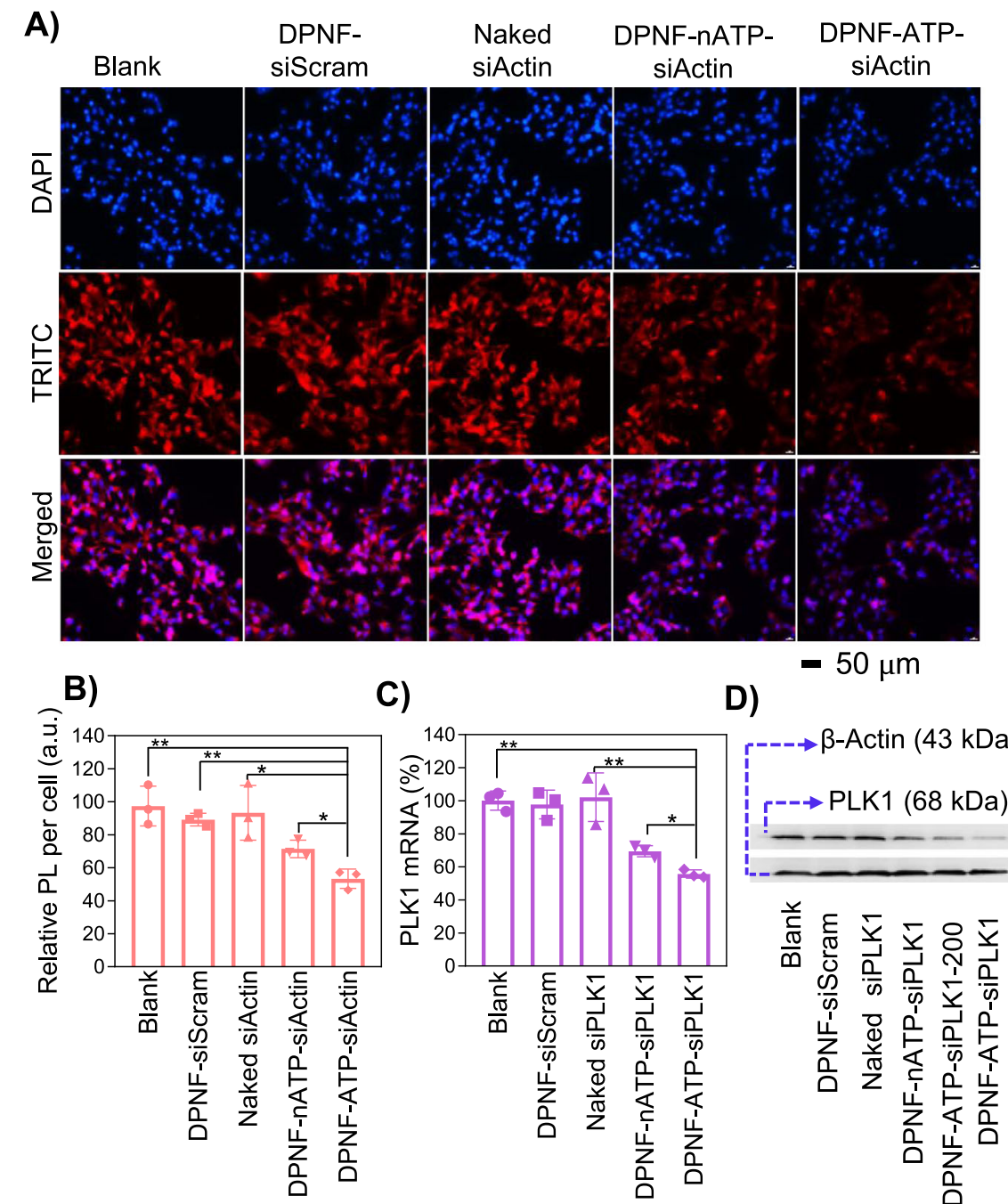

D)
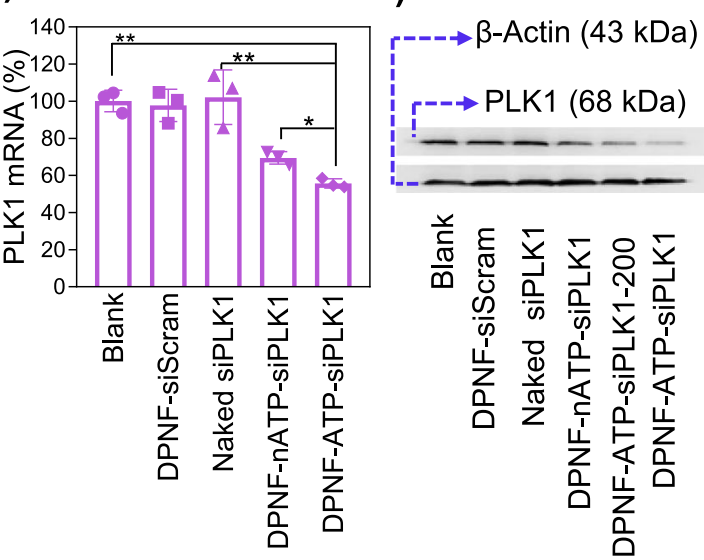

Fig. 6 Gene silencing of siRNA-loaded DPNF-20 in vitro. A Gene silencing effects of siActin-loaded DPNF-20 in MBA-MD-231 cells. MBA-MD-231 cells were treated, respectively, with naked siActin, DPNF-siScram, DPNF-nATP-siActin, and DPNF-ATP-siActin at same dose for $6 \mathrm{~h}$. After $48 \mathrm{~h}$, the cellular actin cytoskeletons were stained with phalloidin-TRITC and observed with fluorescence microscope. The siActin was $300 \mathrm{nM}$, and the DPNF was $420 \mu \mathrm{g} /$ $\mathrm{ml}$. B Quantitative analysis of gene silencing effects of siActin in different formulations in (A). Error bars represent s.d. $\left(n=3\right.$ replicates), ${ }^{\star} p<0.05,{ }^{\star \star} p<$ 0.01. C, D Gene silencing effect of siPLK1-loaded DPNF-20 in MBA-MD-231 cells. C MBA-MD-231 cells were treated, respectively, with naked siPLK1, DPNF-siScram, DPNF-nATP-siPLK1, and DPNF-ATP-siPLK1 with a dose of $300 \mathrm{nM}$ siPLK1 and $420 \mu \mathrm{g} / \mathrm{ml}$ DPNFs. After $48 \mathrm{~h}$, the mRNA levels of PKL1 were measured by RT-qPCR. Error bars represent s.d. ( $n=3$ replicates), ${ }^{\star} p<0.05,{ }^{\star \star} p<0.01$. D MBA-MD-231 cells were treated with naked siPLK1, DPNFsiScram, DPNF-nATP-siPLK1, and DPNF-ATP-siPLK1, respectively, with a dose of $300 \mathrm{nM}$, and DPNF-ATP-siPLK1-200 group was treated with a dose of $200 \mathrm{nM}$ siPLK1 and $420 \mu \mathrm{g} / \mathrm{ml}$ DPNFs. After $48 \mathrm{~h}$, the expression of protein PKL1 was analyzed by western blot.

As demonstrated in vitro, the PBA functional group on SDPNF-20 could react with sialic acid residues over-expressed on MDA-MB-231, to form annular boronate ester and promote the active targeting and accumulation of DPNF-20 in the tumor sites. Although PBA can form $\mathrm{pH}$-responsive borate esters with monosaccharides such as glucose in plasma, PBA could form more stable complex with sialic acid residues even in acidic tumor microenvironment that was lower than its $\mathrm{pKa}$, thus leading to selective and high affinity with tumor cells ${ }^{50,51}$. To demonstrate the targeting ability of DPNF-20 in vivo, MBA-MD-231 cells were planted as subcutaneous xenografts in BALB/c nude mice. Mice were injected intravenously with Cy5-labeled siRNA (Cy5siRNA) and Cy5-siRNA-loaded DPNF-20 (DPNF-Cy5-siRNA), respectively. After $24 \mathrm{~h}$, the mice were sacrificed and the major organs and tumor were collected for ex vivo fluorescence imaging. For the Cy5-siRNA-treated mice, significant fluorescence signals were observed in kidney and liver, but negligible fluorescence signals were observed in the tumor (Fig. 7A). In contrast, for the
DPNF-Cy5-siRNA-treated mice, much stronger fluorescence signals at the tumor site was detected compared with kidney and other organs, exhibiting expressive tumor-targeting ability.

To evaluate the antitumor effect, fifteen mice were randomly divided into five groups and intravenously injected with saline, naked siPLK1, DPNF-siScram, DPNF-nATP-siPLK1, and DPNFATP-siPLK1 once every 2 days, respectively. The siPLK1 dose was $1 \mathrm{mg} / \mathrm{kg}$ per injection. Tumor volumes and body weights of mice were recorded every 2 days, the relative tumor volume was calculated, and the tumor growth and body weight curves with various drug formulation treatments were plotted. Tumor growth curves indicated that the naked siPLK1 and DPNF-siScram groups showed comparable tumor growth with the saline group, while significant tumor suppression was observed in DPNFnATP-siPLK1 and DPNF-ATP-siPLK1 treatment groups with stronger suppression effect in DPNF-ATP-siPLK1 group (Fig. 7B). Body weight curves showed that the mice in all the treatment groups had no weight change compared to the control group 
A)

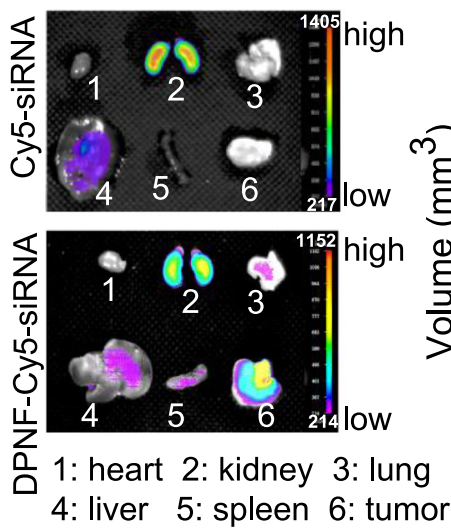

B)

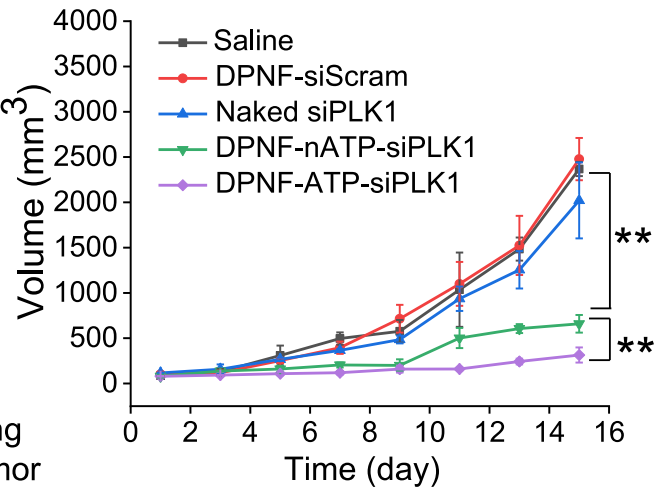

C)

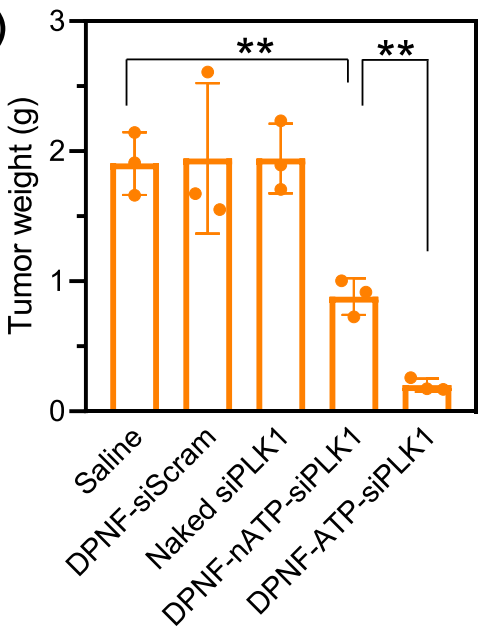

D) DPNF- Naked Saline siScram siPLK1

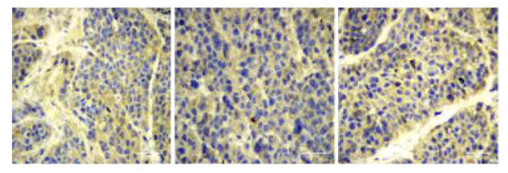

DPNF-nATP DPNF-ATP
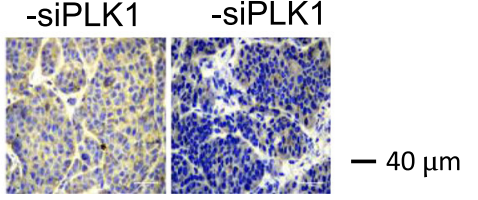

Fig. 7 In vivo targeting ability, antitumor, and gene knockdown effects of siPLK1-loaded DPNF in subcutaneous tumor model. A In vivo targeting, BALB/ c nude mice with subcutaneous xenografts (MDA-MB-231 cells) were injected through tail vein with Cy5-siRNA and DPNF-Cy5-siRNA, respectively. After $24 \mathrm{~h}$, the mice were sacrificed and the tumor, kidney, liver, lung, spleen, and heart were removed for ex vivo imaging. B Tumor growth curves. ${ }^{\star \star} p<0.01$. C Tumor weights measurement. ${ }^{* *} p<0.01$. D Representative immunohistochemical staining of PLK1 protein in tumor tissue in various treatment groups. Data represent mean \pm s.d. $(n=3$ independent mice).

(Supplementary Fig. 16). After 15 days of treatment, the tumor xenografts were excised (Supplementary Fig. 17), and the tumor weights were measured. Compared with saline group, main tumor weight exhibited suppression of $53.68 \%$ and $90.47 \%$, respectively, for DPNF-nATP-siPLK1 and DPNF-ATP-siPLK1 treatment groups (Fig. 7C). Furthermore, the cell apoptosis was analyzed by hematoxylin-eosin (H\&E) staining and the PLK1 protein expression was analyzed by immunohistochemistry. H\&E results showed that both DPNF-nATP-siPLK1 and DPNF-ATPsiPLK1 treatment groups showed patchy necrosis in tumor (Supplementary Fig. 18). Immunohistochemistry analysis showed that, compared with the saline group, the PLK1 protein expression in DPNF-nATP-siPLK1 and DPNF-ATP-siPLK1 treatment groups was downregulated with stronger effect in the DPNF-ATP-siPLK1 treatment group (Fig. 7D). In contrast, the naked siPLK1 and DPNF-siScram treatment did not exhibit significant down-expression of PLK1 in tumor tissues. These results demonstrated that DPNF-ATP-siPLK1 could effectively downregulate the PLK1 expression in vivo given that it could be effectively delivered to tumor sites and released quickly and specifically in cytoplasm.

In conclusion, a DNA HCR-based strategy is developed to achieve spatiotemporally programmable assembly of DNA under nanoconfinement for precise siRNA delivery. In the system, the potential stored in the metastable HCR hairpins succeeded overcoming the steric effect and electrostatic repulsion between the DNA and DPNF to achieve DNA assembly under nanoconfinement and consequently impressive siRNA loading in the DPNF. Moreover, the integration of the unique superiorities of DNA and synthetic polymer overcame the complexity-scalability-error of DNA. By virtue of these features, siRNA loading DPNF with good physiological stability, enhanced cellular uptake, and controlled siRNA release property was obtained; consequently, efficient gene knockdown was achieved both in vitro and in vivo. Given the abundance of DNA-templated reactions and versatile polymer systems, the spatiotemporally programmable assembly of other gene drugs under nanoconfinement is easily achieved, which provides extensible strategy to engineer smart nucleic acid nanoplatform for precision medicine.

\section{Methods}

Materials. $\mathrm{N}$-isopropylacrylamide (NIPAM) and potassium carbonate $\left(\mathrm{K}_{2} \mathrm{CO}_{3}\right)$ were purchased from HEOWNS (Tianjin, China). N, $\mathrm{N}^{\prime}$-Methylenebisacrylamide (Bis), 4-aminophenylboronic acid (4-APBA) and methacryloyl chloride were purchased from Energy Chemical (Shanghai, China). Ammonium persulfate (APS) was purchased from Solarbio Science \& Technology Co., Ltd. (Beijing, China). RPMI 1640 medium, fetal bovine serum (FBS), polyvinylidene fluoride (PVDF) membrane with pore size of $0.22 \mathrm{~mm}$, BCA Protein Assay Kit, total RNA Extraction Kit, 3-(4,5-dimethylthiazol-2-yl)-2,5-diphenyltetrazolium bromide (MTT), and 4',6-diamidino-2-phenylindole (DAPI) were provided by Solarbio Science \& Technology Co., Ltd. (Beijing, China). The primary antibodies PLK1 (208G4) Rabbit mAb (\#4513T), $\beta$-Actin (13E5) Rabbit mAb (\#4970T), and horseradish peroxidase-linked secondary antibody Anti-rabbit IgG, HRP-linked 
antibody (\#7074P2) were purchased from Cell Signaling Technology (Shanghai, China).

DNA and RNA sequences. All DNA oligonucleotides without chemical modifications were synthesized by Shanghai Sangon Biotech Co. Ltd and were purified by polyacrylamide gel electrophoresis. DNA with specially chemical modification was purchased from Shanghai Sangon Biotech Co. Ltd and was purified by HPLC. RNA oligonucleotides were synthesized by Suzhou GenePharma Co. Ltd. All the used oligonucleotide sequences were listed in Supplementary Tables 1 and 2.

Synthesis of 4-((Acroyloxy)methyl) phenylboronic acid (4-MAPBA). $\mathrm{K}_{2} \mathrm{CO}_{3}$ $(2.70 \mathrm{~g}, 20 \mathrm{mmol}, 8.0 \mathrm{eq}$.) was added into water $(5 \mathrm{~mL})$ under magnetic stirring. 4 Aminophenylboronic acid hydrochloride $(0.432 \mathrm{~g}, 2.5 \mathrm{mmol}$, $1.0 \mathrm{eq}$.) was dissolved in acetone $(20 \mathrm{~mL})$ and was added into the re-prepared $\mathrm{K}_{2} \mathrm{CO}_{3}$ aqueous solution. Afterward, methacryloyl chloride $(0.38 \mathrm{~mL}, 5 \mathrm{mmol}, 2.0 \mathrm{eq}$. $)$ was added dropwise into the reaction mixture with magnetic stirring at room temperature. After $40 \mathrm{~h}$ of reaction, the solvent acetone was removed. The left aqueous solution was acidized utilizing $\mathrm{HCl}$ and the product was extracted with ethyl acetate. The ethyl acetate solution was then collected and dried with anhydrous magnesium sulfate. After the ethyl acetate was filtrated and evaporated, precipitation purification was performed to obtain the product. Molecular formula of product: $\mathrm{C}_{9} \mathrm{H}_{11} \mathrm{BNO}_{3}$, yield: $0.17 \mathrm{~g}$, $0.93 \mathrm{mmol}, 38 \%$. The structure of 4-MAPBA was confirmed by the $400 \mathrm{MHz}$ ${ }^{1}$ HNMR (d ${ }^{6}$-DMSO) spectrum (Supplementary Fig. 1).

Preparation of hairpin structures. Defined stoichiometric amounts of DNA H1 or $\mathrm{H} 2$ were added into $1 \times \mathrm{xTE}-\mathrm{Mg}^{2+}$ buffer. The mixtures were heated to $95^{\circ} \mathrm{C}$ for $5 \mathrm{~min}$, annealed to $35^{\circ} \mathrm{C}$ with a rate of $1 \mathrm{~min} /{ }^{\circ} \mathrm{C}$, and then kept at $35^{\circ} \mathrm{C}$ for $2 \mathrm{~min}$ to obtain DNA hairpin structures.

Preparation of Arcydite-DNA. Arcydite-DNA was prepared by mixing equipotent stoichiometric amounts of DNA C1 and C2 in 1xTAE- $\mathrm{Mg}^{2+}$ buffer at room temperature.

Synthesis of DNA cross-linked polymeric nanoframework (DPNF). NIPAM monomer $(65 \mathrm{mM})$, APS initiator (0.1 wt\%), Bis (2 mM), Acry-4-MAPBA (3 mM), and Acrydite-DNA were added into a heart-shaped bottle with continuing $\mathrm{N}_{2}$ bubbling. The reaction solution was heated to $70^{\circ} \mathrm{C}$ under magnetic stirring for 15 $\mathrm{min}$, and the transparent solution changed to milk-white. The synthesized DPNF was collected by centrifugation $(6124 \times g)$ and re-suspended in $1 \times \mathrm{TAE} / \mathrm{Mg}^{2+}$ buffer. The final Acrydite-DNA concentrations used were 5, 10, and $20 \mu \mathrm{M}$ for DPNF-5, DPNF-10, and DPNF-20 preparation, respectively.

Hybridization chain reaction (HCR) of DNA in DPNF. The DPNFs $(4.2 \mathrm{mg} / \mathrm{ml})$ were incubated with $\mathrm{H} 1$ and $\mathrm{H} 2$ (HA) with a final concentration of $3 \mu \mathrm{M}$ at $30^{\circ} \mathrm{C}$ for different time, and then the nanoparticles were collected by centrifugation $(6124 \times g)$

ATP-triggered release of ssDNA-12 from DPNFs. The ssDNA-12-loaded DPNFs were incubated with ATP with a defined concentration at $37^{\circ} \mathrm{C}$ for indicated time. The final concentrations of DPNFs and ssDNA-12 were $4.2 \mathrm{mg} / \mathrm{ml}$ and $3 \mu \mathrm{M}$, respectively. Gel electrophoresis was performed to analyze the release of target nucleic acid ssDNA-12.

Agarose gel electrophoresis. Samples were mixed with loading buffer and analyzed by $1 \%(\mathrm{w} / \mathrm{w})$ agarose with a voltage $6 \mathrm{~V} / \mathrm{cm}$ in TAE buffer. After electrophoresis, the gel was stained by ethidium bromide (EB, $5 \mu \mathrm{g} / \mathrm{ml}$ ), visualized by UV illumination with $312 \mathrm{~nm}$, and photographed by Gel Imaging system.

Polyacrylamide gel electrophoresis (PAGE). Twelve percent native PAGE (acrylamide/N,N'-methylenebisacrylamide $=29: 1$, TBE buffer) was used for sample analysis. After electrophoresis, the gel was stained by EB $(5 \mu \mathrm{g} / \mathrm{ml})$, visualized by UV illumination with $312 \mathrm{~nm}$, and photographed by Gel Imaging system.

SEM characterization. The cleaned silicon wafers were fixed on a SEM sample stage with conductive adhesive. Afterward, samples were dropped on the fixed silicon wafers and placed in a vacuum oven at $35^{\circ} \mathrm{C}$ for $24 \mathrm{~h}$. After drying, the samples were coated with $\mathrm{Au}$, and then were imaged with Hitachi-S4800 FESEM.

Dynamic light scattering (DLS) measurement. The hydration diameter distribution and zeta potential of the DPNFs were characterized by using a Zetasizer Nano ZS90 (Malvern Instruments, Malvern, UK) with $90^{\circ}$ optics and a $\mathrm{He}-\mathrm{Ne}$ Laser $(4.0 \mathrm{~mW}, 633 \mathrm{~nm})$.
Stability assay of DPNF. The stability of DPNF-20 was evaluated by incubating DPNF-20 with $10 \%$ fetal bovine serum (FBS) containing $1 \mathrm{xTAE} / \mathrm{Mg}^{2+}$ for different times at $37^{\circ} \mathrm{C}$, which were followed by analysis with $12 \%$ PAGE. The DNA hairpins were set as $3 \mu \mathrm{M}$, and DPNF-20 was set as $4.2 \mathrm{mg} / \mathrm{ml}$.

Cell culture. The used human breast cancer cell line MDA-MB-231 was cultured in Dulbecco's modified Eagle's medium: $10 \%$ fetal bovine serum contains Nutrient Mixture F-12 (DMEM-F-12) at $37^{\circ} \mathrm{C}$ in a $5 \% \mathrm{CO}_{2}$ contained humidified atmosphere.

Flow cytometry analysis. MDA-MB-231 cells were seeded into 6-well plates and proliferated to around $80-90 \%$ confluence. Culture medium was replaced with fresh medium containing Cy5-labeled DPNFs. The final concentration of Cy5 is 3 $\mu \mathrm{M}$. After incubation for indicated time $(1,2,4$, and $6 \mathrm{~h})$, the cells were washed three times with PBS and harvested by trypsin treatment. Then the harvested cells were washed twice with PBS and collected by centrifugation $(861 \times g$ for $5 \mathrm{~min})$. Finally, the cells were re-suspended with PBS and lifted with a 300 mesh filter for flow cytometry analysis. A figure exemplifying the gating strategy has been included in the Supplementary information (Supplementary Fig. 19).

The 4-boronobenzoic acid (BA) blocking assay. MDA-MB-231 cells were seeded onto a $35-\mathrm{mm}$ glass-bottom dishes at a density of $2 \times 10^{5}$ cells/well and cultured at $37^{\circ} \mathrm{C}$ for $12 \mathrm{~h}$. The medium was replaced with BA $(0.2 \mathrm{mg} / \mathrm{ml})$ contained fresh medium for further incubation of $12 \mathrm{~h}$. Then the medium was replaced with fresh medium containing 5-Carboxytetramethylrhodamine (TAMRA)-labeled DPNF-20 (denoted as TAMRA-SDPNF-20). The concentration of TAMRA-SDPNF-20 was set as $420 \mu \mathrm{g} / \mathrm{ml}$. Subsequently, the cells were washed with PBS three times and imaged with a fluorescence microscope.

Lysosomal escape of DPNFs. MDA-MB-231 cells were seeded onto $35 \mathrm{~mm}$ glassbottom dishes at a density of $2 \times 10^{5}$ cells/well and cultured at $37^{\circ} \mathrm{C}$ for $12 \mathrm{~h}$. The medium was then replaced with $1 \mathrm{ml}$ of fresh medium containing Cy5-labeled DPNF20 (Cy5-DPNF-20). The concentration of Cy5-DPNF-20 was set as $420 \mu \mathrm{g} / \mathrm{ml}$. Intracellular distribution of Cy5-DPNF-20 was analyzed with CLSM. Nuclei and lysosome were, respectively, stained with DAPI and LysoTracker Green dyes.

Cell viability test. The cell viability was evaluated with standard MTT assay. MDA-MB-231 cells were planted on 96-well plates at a density of $5 \times 10^{3}$ cells per well and incubated for $12 \mathrm{~h}$. Afterward, the cells were incubated with DPNF-20 in varied concentrations at $37^{\circ} \mathrm{C}$ for $24 \mathrm{~h}$; then, the cells were washed twice with PBS, and $100 \mu \mathrm{L}$ of MTT solution $(0.5 \mathrm{mg} / \mathrm{ml})$ was added into each well following by a further 4 -h incubation. Finally, the primary medium was removed, and $110 \mu \mathrm{L}$ of DMSO was added. After gently shaking for $10 \mathrm{~min}$, the absorbance at $490 \mathrm{~nm}$ (OD 490) of the wells was measured with a microplate reader.

Real-time quantitative PCR (RT-qPCR) analysis. MDA-MB-231 cells were seeded in six-well plates at a density of $5 \times 10^{5}$ cells per well and incubated for $12 \mathrm{~h}$. Then the cells were treated with naked siPLK1, DPNF-siScram, DPNF-nATPsiPLK1, and DPNF-ATP-siPLK1, respectively. Three hundred nanomolar of siPLK1 was used in the experiments. The concentration of nanoparticles was set as $420 \mu \mathrm{g} / \mathrm{ml}$. Then the cells were washed by PBS and the intracellular total RNA was extracted with a total RNA Extraction Kit and transcribed reversely into cDNA with a FastKing RT Kit (With gDNase). The quantitative PCR analysis was performed by using SuperReal PreMix Plus (SYBR Green). For $\beta$-actin: the forward primer was designed as $5^{\prime}$-ATCGTGCGTGACATTAAGGAGAAG- $3^{\prime}$ and the reverse primer was designed as $5^{\prime}$-AGGAAGGAAGGCTGGAAGAGTG-3'; for PLK1, the forward primer was designed as $5^{\prime}$-GGCAACCTTTTCCTGAATGA- $3^{\prime}$ and the reverse primer was designed as $5^{\prime}$-AATGGACCACACATCCACCT- $3^{\prime}$. The amplification was monitored with LightCycler ${ }^{\circledR} 480$. Data were analyzed with $2^{-\triangle \triangle \mathrm{Ct}}$ method.

Western blot (WB) assay. MDA-MB-231 cells were seeded in six-well plates at a density of $5 \times 10^{5}$ cells per well and incubated for $12 \mathrm{~h}$. Then the cells were, respectively, cultured with naked siPLK1, DPNF-siScram, DPNF-nATP-siPLK1, and DPNF-ATP-siPLK1 with defined concentration of siPLK1 for $6 \mathrm{~h}$. After $48 \mathrm{~h}$, the proteins were extracted with $1 \times$ SDS Lysis Buffer and quantified with BCA Protein Assay Kit. These proteins were diluted into the same concentration, separated by SDS-PAGE gradient gel, and transferred to the PVDF membrane. Then PVDF membranes were blocked in 5\% skimmed milk and incubated with antibodies against PLK1 (1:1000) and $\beta$-actin (1:1000). The membrane was incubated with horseradish peroxidase-linked secondary antibody and then analyzed using an automatic chemiluminescence image system (Tanon 4600SF).

Biosafety assessment. Six mice were equally divided into two groups. One group was treated with DPNF-20 (100 $\left.\mu \mathrm{L}, 8 \mathrm{mg} \cdot \mathrm{mL}^{-1}\right)$ via intravenously injection. Another group was intravenously injected with saline and was set as control group. The body weights of these mice were recorded for 9 days to explore the 
physiological influences of DPNF-20 toward organisms. After the treatment, blood of the mice was collected and tests of blood biochemical parameters were performed in Tianjin Medical University General Hospital (China). Besides, the major organs (heart, liver, and kidney) of the mice were extracted, kept in formaldehyde $(4 \%)$, and stained with hematoxylin and eosin (H\&E) for postmortem histopathology study.

Targeting and distribution in vivo. To study the targeting ability of DPNF in vivo, $\mathrm{BALB} / \mathrm{c}$ nude mice with breast cancer xenografts were injected with $200 \mu \mathrm{l}$ of Cy5labeled naked siRNA (Cy5-siRNA) or Cy5-siRNA-loaded DPNF-20 (DPNF-Cy5siRNA) through tail vein. The concentrations of siRNA and DPNF-20 were $3 \mu \mathrm{M}$ and $4.2 \mathrm{mg} / \mathrm{ml}$, respectively. After $24 \mathrm{~h}$, the ex vivo fluorescent images of organs and tumors were obtained by using Berthold Night OWL LB 983 NC100 Imaging system (Berthold, Germany) with an excitation:emission of 650:700 nm.

Xenograft tumor model. Animal experiments were approved by the ethics committee of Tianjin University in compliance with the law on experimental animals. BALB/c female nude mice (6-week old) were bought from Beijing Huafukang Bioscience Co. Ltd. (Beijing, China). The mice were subcutaneously inoculated with $1 \times 10^{6}$ MDA-MB-231 cells on the right back of the hind leg region and randomly divided into five groups. The control group was intravenously injected with $100 \mu \mathrm{L}$ saline; naked siPLK1, DPNF-siScram, DPNF-nATP-siPLK1, and DPNF-ATP-siPLK1 at equivalent siPLK1 concentration (a dose of $1 \mathrm{mg} / \mathrm{kg}$ ) were intravenously injected via the tail vein at initial every 2 days for continuous five times. DPNF-siScram was DPNF-20 loaded with Scramble RNA; DPNF-nATPsiPLK1 was siPLK1-loaded DPNF-20 without ATP-responsive property; DPNFATP-siPLK1 was siPLK1-loaded DPNF-20 with ATP-responsive property. Tumor volume was calculated according to the following formula:

$$
V=L \times W^{2} \times 0.5
$$

$L$ and $W$ are the longest and shortest diameters of the tumor, respectively.

Statistics and reproducibility. All data were reported as mean \pm standard deviation (s.d.) from at least three independent runs. The ANOVA $F$-test and Student's $t$ test were used to assess the overall among-group and two-group differences, respectively. In all cases, a $p$-value $<0.05$ was considered to be statistically significant. Analyses were performed using Excel2016 analysis software.

Reporting summary. Further information on research design is available in the Nature Research Reporting Summary linked to this article.

\section{Data availability}

All data supporting this manuscript are contained within the main text and Supplementary figures. The data collected and reported in this study are available upon request from the corresponding author (including data presented in the main text and in the Supplementary Information).

Received: 21 September 2020; Accepted: 20 January 2021; Published online: 18 February 2021

\section{References}

1. Mann, S. The origins of life: old problems, new chemistries. Angew. Chem. Int. Ed. 52, 155-162 (2013).

2. Dzieciol, A. J. \& Mann, S. Designs for life: protocell models in the laboratory. Chem. Soc. Rev. 41, 79-85 (2012).

3. Koga, S., Williams, D. S., Perriman, A. W. \& Mann, S. Peptide-nucleotide microdroplets as a step towards a membrane-free protocell model. Nat. Chem. 3, 720-724 (2011).

4. Grommet, A. B., Feller, M. \& Klajn, R. Chemical reactivity under nanoconfinement. Nat. Nanotechnol. 15, 256-271 (2020).

5. Daube, S. S., Bracha, D., Buxboim, A. \& Bar-Ziv, R. H. Compartmentalization by directional gene expression. Proc. Natl Acad. Sci. USA 107, 2836-2841 (2010).

6. Rebek, J. Jr. Molecular behavior in small spaces. Acc. Chem. Res. 42, 1660-1668 (2009).

7. Karzbrun, E., Tayar, A. M., Noireaux, V. \& Bar-Ziv, R. H. Programmable onchip DNA compartments as artificial cells. Science 345, 829-832 (2014).

8. Lucent, D., Vishal, V. \& Pande, V. S. Protein folding under confinement: a role for solvent. Proc. Natl Acad. Sci. USA 104, 10430-10434 (2007).

9. Aufinger, L. \& Simmel, F. C. Artificial gel-based organelles for spatial organization of cell-free gene expression reactions. Angew. Chem. Int. Ed. 57, 17245-17248 (2018)

10. Guo, X. et al. Gene circuit compartment on nanointerface facilitatating cascade gene expression. J. Am. Chem. Soc. 141, 19171-19177 (2019).
11. Guo, X. et al. Architectures produced by PCR-based assembly as gene compartments for cell-free gene-expression reactions. ChemBioChem 20, 2597-2603 (2019)

12. Rubinovich, L. \& Polak, M. The intrinsic role of nanoconfinernent in chemical equilibrium: evidence from DNA hybridization. Nano Lett. 13, 2247-2251 (2013).

13. Downs, A. M., McCallum, C. \& Pennathur, S. Confinement effects on DNA hybridization in electrokinetic micro- and nanofluidic systems. Electrophoresis 40, 792-798 (2019).

14. Yao, C. et al. Double rolling circle amplification generates physically crosslinked DNA network for stem cell fishing. J. Am. Chem. Soc. 142, 3422-3429 (2020).

15. Wang, D. et al. Transformation of biomass DNA into biodegradable materials from gels to plastics for reducing petrochemical consumption. J. Am. Chem. Soc. 142, 10114-10124 (2020).

16. Li, F. et al. Preparation of biomimetic gene hydrogel via polymerase chain reaction for cell-free protein expression. Sci. China Chem. 63, 99-106 (2020).

17. Yuan, Y., Gu, Z., Yao, C., Luo, D. \& Yang, D. Nucleic acid-based functional nanomaterials as advanced cancer therapeutics. Small 15, 1900172 (2019).

18. Tang, J. et al. Super-soft and super-elastic DNA robot with magnetically driven navigational locomotion for cell delivery in confined space. Angew. Chem. Int. Ed. 59, 2490-2495 (2020).

19. Dong, Y. et al. DNA functional materials assembled from branched DNA: design, synthesis, and applications. Chem. Rev. 120, 9420-9481 (2020).

20. Li, F., Tang, J., Geng, J., Luo, D. \& Yang, D. Polymeric DNA hydrogel: design, synthesis and applications. Prog. Polym. Sci. 98, 101163 (2019).

21. Song, P. et al. DNA hydrogel with aptamer-toehold-based recognition, cloaking, and decloaking of circulating tumor cells for live cell analysis. Nano Lett. 17, 5193-5198 (2017).

22. Li, J. et al. Functional nucleic acid-based hydrogels for bioanalytical and biomedical applications. Chem. Soc. Rev. 45, 1410-1431 (2016).

23. Liu, J., Wang, Z., Zhao, S. \& Ding, B. Multifunctional nucleic acid nanostructures for gene therapies. Nano Res. 11, 5017-5027 (2018).

24. Zhao, Y., Chen, F., Li, Q., Wang, L. \& Fan, C. Isothermal amplification of nucleic acids. Chem. Rev. 115, 12491-12545 (2015).

25. Liao, W.-C. et al. pH- and ligand-induced release of loads from DNAacrylamide hydrogel microcapsules. Chem. Sci. 8, 3362-3373 (2017).

26. Fern, J. \& Schulman, R. Modular DNA strand-displacement controllers for directing material expansion. Nat. Commun. 9, 3766 (2018).

27. Wijnands, S. P. W., Meijer, E. W. \& Merkx, M. DNA-functionalized supramolecular polymers: dynamic multicomponent assemblies with emergent properties. Bioconj. Chem. 30, 1905-1914 (2019).

28. Ouyang, C. et al. Precision-guided missile-like DNA nanostructure containing warhead and guidance control for aptamer-based targeted drug delivery into cancer cells in vitro and in vivo. J. Am. Chem. Soc. 142, 1265-1277 (2020).

29. Cutler, J. I., Auyeung, E. \& Mirkin, C. A. Spherical nucleic acids. J. Am. Chem. Soc. 134, 1376-1391 (2012).

30. Liu, K., Zheng, L., Ma, C., Goestl, R. \& Herrmann, A. DNA-surfactant complexes: self-assembly properties and applications. Chem. Soc. Rev. 46, 5147-5172 (2017)

31. Chidchob, P., Edwardson, T. G. W., Serpell, C. J. \& Sleiman, H. F. Synergy of two assembly languages in DNA nanostructures: self-assembly of sequencedefined polymers on DNA cages. J. Am. Chem. Soc. 138, 4416-4425 (2016).

32. Tokura, Y. et al. Bottom-up fabrication of nanopatterned polymers on DNA origami by in situ atom-transfer radical polymerization. Angew. Chem. Int. Ed. 55, 5692-5697 (2016).

33. Tokura, Y. et al. Polymer tube nanoreactors via DNA-origami templated synthesis. Chem. Commun. 54, 2808-2811 (2018).

34. Hu, Y. et al. A shape memory acrylamide/DNA hydrogel exhibiting switchable dual pH-responsiveness. Adv. Funct. Mater. 25, 6867-6874 (2015).

35. Kahn, J. S., Hu, Y. \& Willner, I. Stimuli-responsive DNA-based hydrogels: from basic principles to applications. Acc. Chem. Res. 50, 680-690 (2017).

36. Cangialosi, A. et al. DNA sequence-directed shape change of photopatterned hydrogels via high-degree swelling. Science 357, 1126-1129 (2017).

37. Ding, F. et al. A crosslinked nucleic acid nanogel for effective sirna delivery and antitumor therapy. Angew. Chem. Int. Ed. 57, 3064-3068 (2018).

38. Zhu, G. et al. Building fluorescent DNA nanodevices on target living cell surface. Angew. Chem. Int. Ed. 52, 5490-5496 (2013).

39. Bi, S., Yue, S. \& Zhang, S. Hybridization chain reaction: a versatile molecular tool for biosensing, bioimaging, and biomedicine. Chem. Soc. Rev. 46, 4281-4298 (2017).

40. Mo, R., Jiang, T., DiSanto, R., Tai, W. \& Gu, Z. ATP-triggered anticancer drug delivery. Nat. Commun. 5, 3364 (2014).

41. Biswas, S. et al. Biomolecular robotics for chemomechanically driven guest delivery fuelled by intracellular ATP. Nat. Chem. 5, 613-620 (2013).

42. Xiao, M. et al. Programming drug delivery kinetics for active burst release with DNA toehold switches. J. Am. Chem. Soc. 141, 20354-20364 (2019). 
43. Yoshinaga, N. et al. Polyplex micelles with phenylboronate/gluconamide cross-linking in the core exerting promoted gene transfection through spatiotemporal responsivity to intracellular $\mathrm{pH}$ and ATP concentration. J. Am. Chem. Soc. 139, 18567-18575 (2017).

44. Lamping, S., Otremba, T. \& Ravoo, B. J. Carbohydrate-responsive surface adhesion based on the dynamic covalent chemistry of phenylboronic acidand catechol-containing polymer brushes. Angew. Chem. Int. Ed. 57, 2474-2478 (2018).

45. Zhao, X. et al. Co-delivery of HIF1 alpha siRNA and gemcitabine via biocompatible lipid-polymer hybrid nanoparticles for effective treatment of pancreatic cancer. Biomaterials 46, 13-25 (2015).

46. Whitehead, K. A., Langer, R. \& Anderson, D. G. Knocking down barriers: advances in siRNA delivery. Nat. Rev. Drug Discov. 8, 129-138 (2009).

47. Zhao, H. et al. Persistent luminescent nanoparticles containing hydrogels for targeted, sustained, and autofluorescence-free tumor metastasis imaging. Nano Lett. 20, 252-260 (2020).

48. Wang, D. et al. A molecular recognition approach to synthesize nucleoside analogue based multifunctional nanoparticles for targeted cancer therapy. J. Am. Chem. Soc. 139, 14021-14024 (2017).

49. Mou, Q. et al. Two-in-one chemogene assembled from drug-integrated antisense oligonucleotides to reverse chemoresistance. J. Am. Chem. Soc. 141, 6955-6966 (2019).

50. Deshayes, S. et al. Phenylboronic acid-installed polymeric micelles for targeting sialylated epitopes in solid tumors. J. Am. Chem. Soc. 135, 15501-15507 (2013).

51. Long, Y. et al. Enhanced melanoma-targeted therapy by "Fru-blocked" phenyboronic acid-modified multiphase antimetastatic micellar nanoparticles. Adv. Sci. 5, 1800229 (2018).

\section{Acknowledgements}

This work was supported in part by National Natural Science Foundation of China (Grant Nos.: 31971305, 21905196, and 21621004), Ministry of Science and Technology of China (National Key Technology Research and Development Program, Grant Nos.: 2019YFA09005800 and 2018YFA0902300), and Tianjin Natural Science Foundation (Basic research plan, Grant Nos: 18JCJQJC47600 and 19JCQNJC01900). We thank Taotao Li, Huaixin Zhao, and Chi Yao at Tianjin University for their help on experiments and discussion.

\section{Author contributions}

F.L. and D.Y. designed the experiments. F.L., W.Y. and J.Z. conducted the experiments. F.L., W.Y., J.Z., Y.D., X.D., X.R., Z.G. and D.Y. analyzed data and wrote the manuscript. D.Y. supervised the project.

\section{Competing interests}

The authors declare no competing interests.

\section{Additional information}

Supplementary information The online version contains supplementary material available at https://doi.org/10.1038/s41467-021-21442-7.

Correspondence and requests for materials should be addressed to D.Y.

Peer review information Nature Communications thanks the anonymous reviewer(s) for their contribution to the peer review of this work.

Reprints and permission information is available at http://www.nature.com/reprints

Publisher's note Springer Nature remains neutral with regard to jurisdictional claims in published maps and institutional affiliations.

(c) (i) Open Access This article is licensed under a Creative Commons Attribution 4.0 International License, which permits use, sharing, adaptation, distribution and reproduction in any medium or format, as long as you give appropriate credit to the original author(s) and the source, provide a link to the Creative Commons license, and indicate if changes were made. The images or other third party material in this article are included in the article's Creative Commons license, unless indicated otherwise in a credit line to the material. If material is not included in the article's Creative Commons license and your intended use is not permitted by statutory regulation or exceeds the permitted use, you will need to obtain permission directly from the copyright holder. To view a copy of this license, visit http://creativecommons.org/ licenses/by/4.0/.

(C) The Author(s) 2021 